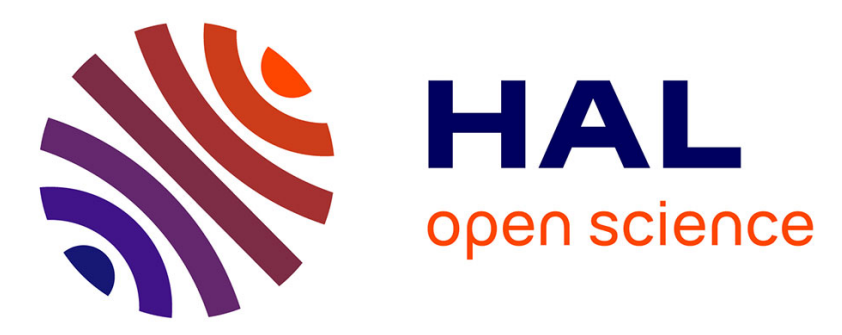

\title{
Numerical investigation of polarization filtering for direct optical imaging within scattering media
}

Callum A Macdonald, Ugo Tricoli, Anabela da Silva, Vadim A. Markel

\section{To cite this version:}

Callum A Macdonald, Ugo Tricoli, Anabela da Silva, Vadim A. Markel. Numerical investigation of polarization filtering for direct optical imaging within scattering media. Journal of the Optical Society of America. A Optics, Image Science, and Vision, 2017, 34 (8), pp.1330. 10.1364/JOSAA.34.001330 . hal-02376520

\section{HAL Id: hal-02376520 \\ https://hal.science/hal-02376520}

Submitted on 26 Nov 2019

HAL is a multi-disciplinary open access archive for the deposit and dissemination of scientific research documents, whether they are published or not. The documents may come from teaching and research institutions in France or abroad, or from public or private research centers.
L'archive ouverte pluridisciplinaire HAL, est destinée au dépôt et à la diffusion de documents scientifiques de niveau recherche, publiés ou non, émanant des établissements d'enseignement et de recherche français ou étrangers, des laboratoires publics ou privés. 


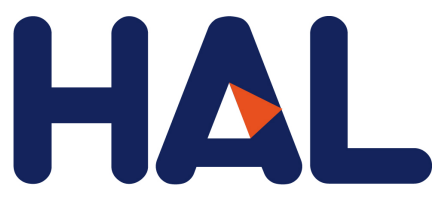

archives-ouvertes

\section{Numerical investigation of polarization filtering for direct optical imaging within scattering media}

Callum Macdonald, Ugo Tricoli, Anabela da Silva, Vadim A. Markel

\section{To cite this version:}

Callum Macdonald, Ugo Tricoli, Anabela da Silva, Vadim A. Markel. Numerical investigation of polarization filtering for direct optical imaging within scattering media. Journal of the Optical Society of America. A Optics, Image Science, and Vision, Optical Society of America, 2017, 34 (8), pp.1330. 10.1364/JOSAA.34.001330 . hal-02376520

\section{HAL Id: hal-02376520 \\ https://hal.archives-ouvertes.fr/hal-02376520}

Submitted on 26 Nov 2019

HAL is a multi-disciplinary open access archive for the deposit and dissemination of scientific research documents, whether they are published or not. The documents may come from teaching and research institutions in France or abroad, or from public or private research centers.
L'archive ouverte pluridisciplinaire HAL, est destinée au dépôt et à la diffusion de documents scientifiques de niveau recherche, publiés ou non, émanant des établissements d'enseignement et de recherche français ou étrangers, des laboratoires publics ou privés. 


\author{
Callum M. Macdonald, ${ }^{1, *}$ Ugo Tricoli, ${ }^{1}$ Anabela Da Silva, ${ }^{1}$ and Vadim A. Markel ${ }^{1,2}$ (d) \\ ${ }^{1}$ Aix Marseille Univ, CNRS, Centrale Marseille, Institut Fresnel, F-13013 Marseille, France \\ ${ }^{2}$ Department of Radiology, University of Pennsylvania, Philadelphia, Pennsylvania 19104, USA \\ *Corresponding author: callum.macdonald@fresnel.fr
}

Received 7 April 2017; revised 13 June 2017; accepted 27 June 2017; posted 28 June 2017 (Doc. ID 292246); published 0 MONTH 0000

\begin{abstract}
We investigate the ability of polarization filtering to improve direct imaging of absorbing objects which are buried within scattering environments. We extend on previous empirical investigations by exploiting an efficient perturbation-based formalism, which is applicable to arbitrarily arranged sources and detectors with arbitrary polarizations. From this approach, we are able in some cases to find certain non-trivial linear combinations of polarization measurement channels that maximize the object resolution and visibility. OCIS codes: (110.6960) Tomography; (290.5855) Scattering, polarization; (110.5405) Polarimetric imaging.
\end{abstract}

https://doi.org/10.1364/JOSAA.99.099999

\section{INTRODUCTION}

Analysis of diffuse, multiply scattered light propagating through turbid media $[1,2]$ has long been used in optical tomography to non-invasively retrieve information about optical properties of interest, such as the three-dimensional distributions of the absorption and scattering coefficients [3-5]. Neglecting the effects of phase and interference, multiply scattered light can be described by the radiative transport equation (RTE) or, at a less fundamental level, by the diffusion equation, which is an approximation to the former. Optical tomography frequently relies on the diffusion equation (e.g., $[6,7]$ ) since inversion of the RTE is a complicated mathematical task. There exists, however, a persistent interest in imaging through turbid media in the mesoscopic scattering regime or beyond the limitations of the diffusion approximation, which include the requirement that the scattering be much stronger than absorption, the requirement of sufficiently large source-detector separations, and neglect of light polarization. If these conditions are not met, the diffusion approximation is inapplicable and the RTE must be used instead.

Many numerical and analytical approaches to solving the RTE have been explored in the past. The commonly used numerical methods are based on using discrete ordinates for the angular variable and discrete difference or finite element discretization for the spatial variable, or on Monte Carlo simulations. The advantage of numerical methods is generality and accurate handling of the medium boundaries while the disadvantage is high, often unmanageable computational complexity. Therefore, several analytical methods for solving the RTE have been developed. Cumulant expansion of the time-dependent RTE Green's function was proposed in [8] for an infinite medium. Later this method was generalized to account for light polarization [9] and to media with planar boundaries [10]. In [10], the sensitivity kernel (the weight function) of linearized optical tomography has also been computed. However, calculation of the cumulant expansion past second order is rather complicated. In addition, the boundary conditions used in [10] are appropriate for the diffuse propagation regime but not compatible mathematically with the more complicated half-range RTE boundary conditions. A different analytical approach based on the method of rotated reference frames (MRRF) was developed in [11-13]. In the MRRF, there is no restriction on the order of expansion and the rigorous half-range boundary conditions of the RTE are used. However, the limitation is that the MRRF constructs an expansion in a finite orthonormal basis of functions that are not square-integrable. This results in numerical instabilities. Although this problem was rectified in later research [14], it can be concluded that numerical methods and, in particular, Monte Carlo simulations retain their significance and utility in the mesoscopic scattering regime.

In this paper, we utilize a recently developed Monte Carlobased method for computing the sensitivity kernels of optical tomography [15]. With the improvements described in [15], we can perform such calculations with sufficient efficiency. The main goal of the simulations reported below is to show how polarization filtering and computational post-processing of data can be used to improve the visibility of objects buried within multiply scattering environments directly, without solving a complicated inverse problem.
45

46

47

48

49

50

51

52

53

54

55

56

57

58

59

60

61

62

63

64

65

66

67

68

69

70

71

72

73

74 
So far, there have been few studies of polarization filtering in tomography. Inversion of the RTE has been reported using both numerical [16-18] and analytical [19] methods. However, the vast majority of these implementations, and all those exploiting the diffusion equation, are limited to the use of unpolarized light and polarization-insensitive measurements. We note that the standard diffusion equation does not account for polarization of light. Generalization of the diffusion theory for polarized light can potentially be considered by computing the first two angular moments of the vector RTE (vRTE). However, this approach appears to be problematic due to the very nature of the diffusion approximation: it assumes that the light at any given point in space is almost isotropic, yet the spatial regions where this condition is satisfied also tend to be vacant of any preferred orientation of linear polarization [20]. There are additional difficulties related to computing angular moments of functions involving four-dimensional Stokes vectors, which must be defined in a special reference frame for each propagation direction, and a large number of coupled equations that can be formally derived by this approach. It appears that attempts to use the diffusion theory to describe polarization would be impractical for all but the circular component of polarization, which may persist long after isotropization of photon direction in certain media $[21,22]$. On the other hand, the vRTE is sufficiently flexible and contains a description of polarization that is adequate for the purposes of imaging.

The fact that polarization and the vRTE are rarely exploited in optical tomography could be thought of as a shortcoming given that in the context of non-tomographic imaging in turbid media, it is well established that the use of polarized light can remove some of the blurring effects of multiple scattering. This has been demonstrated for imaging through a variety of scattering media, such as fog [23], water [24], and tissues [25]. Crucial for direct imaging is the extraction of ballistic light and other "short-path" photons from the diffuse background. Correspondingly, numerous gating techniques have been proposed in order to extract the non-diffuse photons.

Schmitt et al. [26] showed that short-path photons can be extracted through subtraction of two orthogonal polarization measurements. They then demonstrated increased image contrast for 1D scanning of absorbing objects. Similarly, Emile et al. [27] selected polarization-maintaining photons through polarization modulation and obtained 1D profiles of embedded objects. Following the same ideas, Mujumdar and Ramachandran [28] improved the experimental setup, generating $2 \mathrm{D}$ images on a CCD camera without the need to scan. Demos and Alfano [25] have shown the usefulness of considering separately the parallel and perpendicular polarization components of light pulses in backscattering. Silverman and Strange [29] found an increase in visibility when imaging objects through a scattering medium composed of latex spheres in water. They also showed better image contrast with circular polarization compared to linear polarization. Likewise, Lewis et al. [30] reported increased target visibility for circular polarization when imaging through polystyrene sphere suspensions. $\mathrm{Ni}$ et al. used time-gating and early-arriving photon detection to improve the information content in the state of polarization of light passing through turbid media [31] with the application to wireless communication through the atmosphere. More recently, Miller et al. [32] used circular polarization for imaging through fog. Da silva et al. used elliptically polarized light to vary imaging depth [33]. Further, Sridhar and Da Silva [34] investigated the use of elliptically polarized channels to increase imaging contrast in tissues.

The above-mentioned works have demonstrated experimentally the deblurring effect of polarization gating. However, far fewer theoretical investigations of polarization gating have been performed. In one such study, Tyo [35] has calculated analytically and numerically the point spread function of linearly polarized light (at the detector in the transmission geometry). It was shown that a significant narrowing of the point spread function was achieved when the difference of the field components is considered instead of their sum. Later work by Moscoso et al. showed similar findings, and also highlighted the dependence on the type of scattering material [36].

In this work we seek a deeper understanding of the effects of polarization gating for direct imaging by combining some elements and mathematical approaches that are used in tomographic modalities of optical imaging with polarization-gating techniques that are employed in non-tomographic imaging. In particular, we utilize the recently developed numerical technique [15], which allows one to efficiently compute the sensitivity kernel for polarization-resolved optical tomography by Monte Carlo simulations. This provides insight into the effect of perturbations at all locations within a medium. We then investigate various physical polarization gates or linear combinations thereof and find those that result in the strongest deblurring of images in both transmission and reflection geometries. We show that the combinations of polarization measurement channels which maximize the image quality are at times non-trivial and depend on the type of media and imaging geometry.

We stress that the scanning-based approach to imaging of turbid media demonstrated in this study does not require solution of an ill-posed inverse problem and is in this sense direct. Yet, while our approach is not tomographic, we make a step toward defining sensitivity kernels that are the least ill-posed and, therefore, most conductive for performing tomographic reconstructions.

The remainder of this paper is organized as follows. In Section 2 we define the sensitivity kernel for polarizationselective optical tomography. In Section 3 we consider scanning of a plane-parallel sample by an axially aligned source-detector pair in the transmission geometry. Reflection geometry is further considered in Section 4. The summary and discussion are given in Section 5.

\section{POLARIZATION-DEPENDENT SENSITIVITY KERNEL}

Consider a slab of scattering material occupying the region $0<z<L$. Inside the medium, the vector specific intensity $\mathcal{I}(\mathbf{r}, \hat{\mathbf{s}})=(I, Q, U, V)$ describing the four Stokes components obeys the stationary vRTE [37]. Here, $\mathbf{r}$ is the vector of position, $\hat{\mathbf{S}}$ is a unit vector specifying the direction in space, and all four Stokes components are functions of these two variables, with dimensionality of power per surface area per unit solid 
angle. Below, we will use calligraphic capital letters to denote various four-component vectors, such as the Stokes vector of specific intensity.

Referring to Fig. 1, let a collimated, continuous-wave laser source described by $\delta\left(\mathbf{r}-\mathbf{r}_{a}\right) \delta\left(\hat{\mathbf{s}}-\hat{\mathbf{s}}_{a}\right) \mathcal{S}_{\text {in }}$ be incident on the medium, where $\mathcal{S}_{\text {in }}$ is a vector describing an arbitrary polarization state of the source. Note that $\mathcal{S}_{\text {in }}$ has the dimensionality of power. A detector then measures the specific intensity of light exiting the medium at a different point on the slab surface, $\mathbf{r}_{b}$, and in the direction of $\hat{\mathbf{s}}_{b}$. We will explore both the transmission and reflection geometries, which are illustrated in Figs. 1(a) and 1(b), respectively. Further, we may place a polarization filter in front of the detector whose effect on the specific intensity can be characterized by the projection onto a dimensionless output state vector $\mathcal{S}_{\text {out }}$. If we define this state vector to have unit magnitude, then the measured signal is the scalar product $\frac{1}{2} \mathcal{S}_{\text {out }} \cdot \mathcal{I}\left(\mathbf{r}_{b}, \hat{\mathbf{s}}_{b}\right)$.

If the medium is not spatially uniform, the measured signal will depend on the location and strength of any inhomogeneities that are present. We assume that the inhomogeneities are purely absorbing so that we can write for the scattering and absorption coefficients of the medium $\mu_{s}(\mathbf{r})=\bar{\mu}_{s}$ and $\mu_{a}(\mathbf{r})=\bar{\mu}_{a}+\delta \mu_{a}(\mathbf{r})$, where $\bar{\mu}_{s}$ and $\bar{\mu}_{a}$ are constant background values of the respective coefficients and $\delta \mu_{a}(\mathbf{r})$ is the absorptive inhomogeneity. We further assume that $\delta \mu_{a}(\mathbf{r})$ is
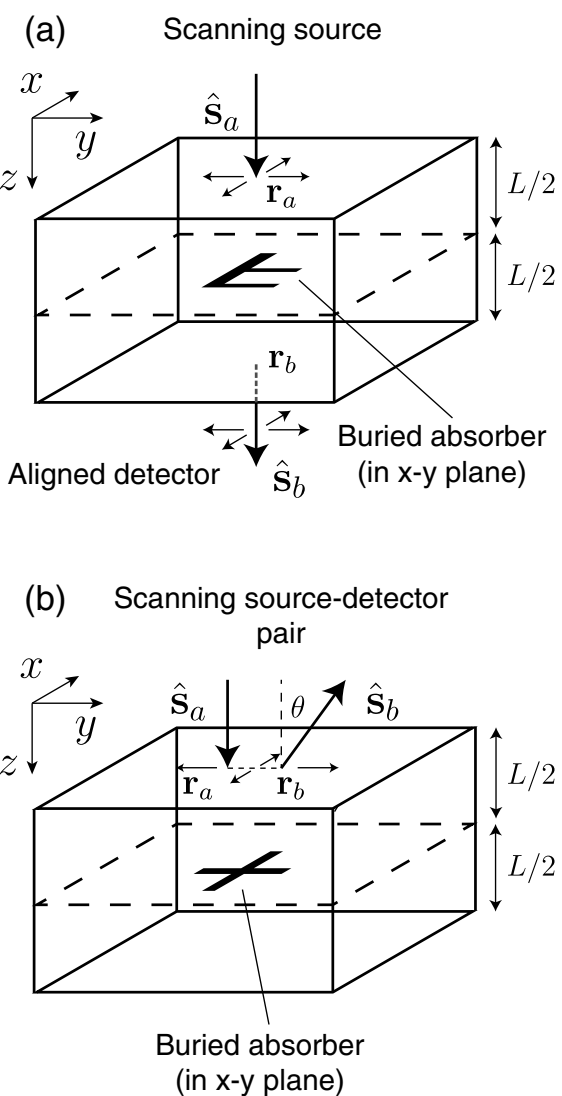

F1:1 Fig. 1. Geometries of scanned images of buried absorbers. (a) OnF1:2 axis transmission geometry with normally aligned source and detector. F1:3 (b) Backscattering using a scanning source-detector pair separated by a F1:4 distance $\left|\mathbf{r}_{a b}\right|$ in the $Y$ direction. Source is normally incident while the F1:5 detector collects light in the direction $\hat{\mathbf{s}}_{b}$. sufficiently small, either in magnitude or in its support, so that the vRTE can be linearized in $\delta \mu_{a}$. Then, within the accuracy of the first Born approximation, we have

$$
\mathcal{I}\left(\mathbf{r}_{b}, \hat{\mathbf{s}}_{b}\right)=\mathcal{I}_{0}\left(\mathbf{r}_{b}, \hat{\mathbf{s}}_{b}\right)-\int K\left(\mathbf{r}_{b}, \hat{\mathbf{s}}_{b}, \mathbf{r}_{a}, \hat{\mathbf{s}}_{a} ; \mathbf{r}\right) \mathcal{S}_{\text {in }} \delta \mu_{a}(\mathbf{r}) \mathrm{d}^{3} r,
$$

where

$$
K\left(\mathbf{r}_{b}, \hat{\mathbf{s}}_{b}, \mathbf{r}_{a}, \hat{\mathbf{s}}_{a} ; \mathbf{r}\right)=\int G_{0}\left(\mathbf{r}_{b}, \hat{\mathbf{s}}_{b} ; \mathbf{r}, \hat{\mathbf{s}}\right) G_{0}\left(\mathbf{r}, \hat{\mathbf{s}} ; \mathbf{r}_{a}, \hat{\mathbf{s}}_{a}\right) \mathrm{d}^{2} s
$$

is the sensitivity kernel of optical tomography [15], a quantity that is central to imaging. Also, $G_{0}\left(\mathbf{r}, \hat{\mathbf{s}} ; \mathbf{r}^{\prime}, \hat{\mathbf{s}}^{\prime}\right)$ is the $4 \times 4$ Green's function for the vRTE in the homogeneous reference medium with $\delta \mu_{a}=0$ and, finally, $\mathcal{I}_{0}$ is the specific intensity in the reference medium. For the latter quantity, we can write

$$
\mathcal{I}_{0}\left(\mathbf{r}_{b}, \hat{\mathbf{s}}_{b}\right)=G_{0}\left(\mathbf{r}_{b}, \hat{\mathbf{s}}_{b} ; \mathbf{r}_{a}, \hat{\mathbf{s}}_{a}\right) \mathcal{S}_{\text {in }} .
$$

We can now define the $4 \times 4$ matrix data function $\Phi$ by the relation

$$
\Phi \mathcal{S}_{\text {in }} \equiv \mathcal{I}_{0}-\mathcal{I} \text {. }
$$

This data function depends on the positions and collimation directions of the source and detector, that is, $\Phi=\Phi\left(\mathbf{r}_{b}, \hat{\mathbf{s}}_{b} ; \mathbf{r}_{a}, \hat{\mathbf{s}}_{a}\right)$. We then have the following equation coupling the inhomogeneities of the medium to the data function:

$$
\int K\left(\mathbf{r}_{b}, \hat{\mathbf{s}}_{b}, \mathbf{r}_{a}, \hat{\mathbf{s}}_{a} ; \mathbf{r}\right) \delta \mu_{a}(\mathbf{r}) \mathrm{d}^{3} r=\Phi\left(\mathbf{r}_{b}, \hat{\mathbf{s}}_{b} ; \mathbf{r}_{a}, \hat{\mathbf{s}}_{a}\right),
$$

which is a generalization of the linearized equation of optical tomography that was derived in [38] for the scalar RTE. The most obvious difference here is that both $K$ and $\Phi$ are now matrices. While the first element $\Phi_{11}$ has the same interpretation as in the scalar problem (as the shadow in the measured intensity created by the absorptive heterogeneities), the remaining elements contain additional information. The scalar quantity $\frac{1}{2} \mathcal{S}_{\text {out }} \cdot \Phi \mathcal{S}_{\text {in }}$ is the difference between the physical measurement channels recorded for the homogeneous and the perturbed medium. Individually, these channels are positive scalars for any physically accessible states $\mathcal{S}_{\text {in }}$ and $\mathcal{S}_{\text {out }}$, and the subtraction of two such measurements (resulting in the shadow $\frac{1}{2} \mathcal{S}_{\text {out }} \cdot \Phi \mathcal{S}_{\text {in }}$ ) corresponds to various linear combinations of the elements of $\Phi$. Note that, while these individual measurements are positive scalars, the elements of $\Phi$ can be positive or negative [15]. Another substantial difference is that, in the scalar problem, the diffusion approximation can be introduced in the appropriate limit, significantly simplifying the computation of the sensitivity kernel [39]. In contrast, a diffusion limit for Eq. (5) is not known and there are technical difficulties in deriving a diffusion approximation for this quantity. In addition, we wish to exploit the information contained in the additional elements of $\Phi$, which are most significant in the sub-diffusion regime. In this case, $K$ can be computed by solving vRTE for a given reference medium and $\Phi$ (or some linear combination of its elements) can be obtained by performing several physical measurements.

In what follows, we use Monte Carlo simulations and the technique developed in [15] to compute the Green's function 
(Rayleigh)

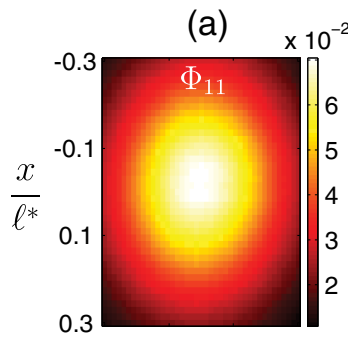

(e)

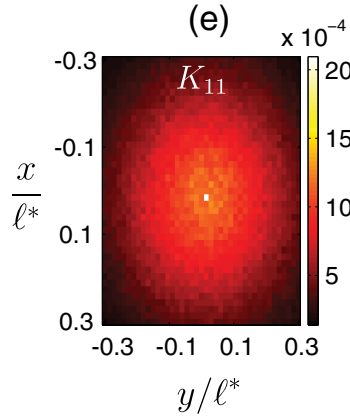

(b)

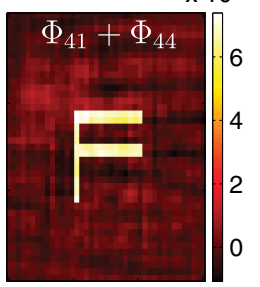

(f)

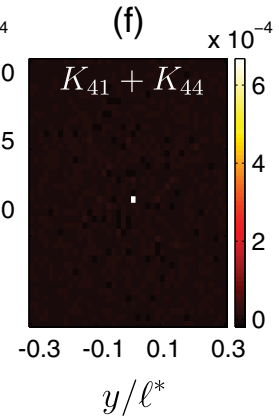

(Mie)

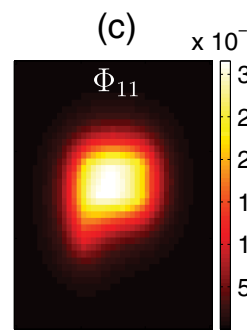

(g)

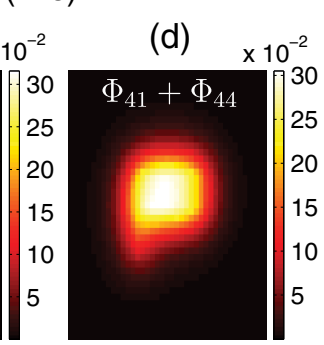

(h)

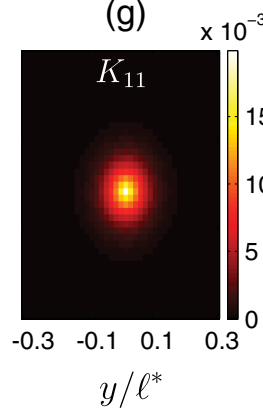

Fig. 2. Sensitivity to a buried object within a Rayleigh scattering medium of optical depth $L=20 \ell^{*}$ [first two columns, Panels (a),(b),(e),(f)] and within a Mie scattering medium of optical depth $L=1 \ell^{*}$ [second two columns, Panels (c),(d),(g),(h)]. The physical depth of both media is the same and equal to $20 / \bar{\mu}_{t}$. The top row of images [Panels (a)-(d)] display various matrix elements of the data function $\Phi$ obtained by scanning the sourcedetector pair in the $X Y$-plane for a buried object shaped as the letter "F." The second row of images [Panels (e)-(h)] show the corresponding matrix elements of the sensitivity kernel $K$ within the object plane, at $z=L / 2$, for a single, centrally located position of the source and detector. All figures are normalized to their respective kernel element $K_{11}$ summed over the entire object plane.

$G_{0}$ and the sensitivity kernel $K$ numerically. We then substitute some "objects," that is, model functions $\delta \mu_{a}(\mathbf{r})$ into Eq. (5) and compute the dependence of the data function on the positions of the source and detector. The advantage of this approach is that, once $K$ is computed for a given medium and imaging geometry, we can simulate easily any individual measurement channel or a combination of such channels. In this way, we can, in some cases, obtain the image of $\delta \mu_{a}(\mathbf{r})$ directly, that is, without inverting Eq. (5). To achieve this result, we will examine which matrix element of $K$ (or a linear combination thereof) is closest to a delta function at the location of interest within the medium and, therefore, provides the sharpest point spread function for imaging. This result, in turn, informs us of which physical measurement channels and which post-processing should be used to achieve the greatest resolution of a buried object at a given location.

\section{SCANNING WITH ALIGNED SOURCE AND DETECTOR IN TRANSMISSION GEOMETRY}

In this section, we use Monte Carlo simulations to compute the sensitivity kernel and the data function in the transmission geometry for the case when the source and detector are aligned directly on axis. Referring to Fig. 1(a), a collimated laser light source is normally incident on the upper surface of the medium, and a detector is arranged to collect light leaving the slab on axis to the source at the lower surface. This source-detector pair is scanned in the $X Y$-plane. Note that this data collection scheme is not equivalent to wide-front illumination of the medium and taking a photograph of the other side. The target is a purely absorbing planar object located in the mid-plane of the slab. In the simulations, the width of the target in the $Z$ direction was equal to one voxel used for accumulating the Monte Carlo statistics. We emphasize that no depth resolution is obtained or sought in these simulations.

Figures 2(a)-2(d) show the dependence of the data function $\Phi$ on the lateral position $(x, y)$ of the source-detector pair for an object shaped as the letter "F," and Figs. 2(e)-2(h) show the dependence of the matrix kernel $K$ on $(x, y)$ for $z=L / 2$ with the source-detector axis being fixed at the center of the field of view. The two left columns [Panels (a), (b), (e), (f)] correspond to a medium containing Rayleigh scattering particles (the scattering asymmetry parameter is $g=0$ in this case) with an optical depth of $L=20 \ell^{*}$, where $\ell^{*}=1 /\left[\bar{\mu}_{a}+(1-g) \bar{\mu}_{s}\right]$ is the transport mean free path. The right two columns [Panels (c), (d), (g), (h)] are for a medium containing large spherical particles (which we will refer to as "Mie" particles) with highly forward-peaked scattering characterized by the asymmetry parameter $g=0.95$, and a slab depth of $L=1 \ell^{*}$. We note that the single scattering matrix of such particles can be computed from Mie theory, given their size, and refractive index relative to the background medium [40]. The Mie particles in this example have a size parameter $k a=7.15$, where $k$ is the wavenumber in the background material of the slab and $a$ is the particle radius. Further, the refractive index contrast is $n_{s} / n_{b}=1.037$, where $n_{s}$ and $n_{b}$ are the sphere and the background refractive indices, respectively. Note that the physical thickness of both media is the same and is equal to $20 / \bar{\mu}_{t}$. 
Here $\bar{\mu}_{t}=\bar{\mu}_{a}+\bar{\mu}_{s}$ is the attenuation coefficient, which is assumed to be the same in both cases. Additionally, the scattering albedo is $\bar{\mu}_{s} / \bar{\mu}_{t}=0.99$ in both media. Thus, the effects of absorption do not play a significant role in this study. We note, however, that stronger values of absorption (a smaller albedo) can result in a similar deblurring effect, as was demonstrated in [41].

The various images in Fig. 2 correspond to different linear combinations of the elements of $\Phi$ and $K$, where all such elements have been normalized to the sum of $K_{11}$ over the entire object plane. The $\Phi_{11}$ element represents the difference in intensity (the shadow) between the case where the absorber is present and the case of homogeneous medium, given an unpolarized illumination and an unfiltered detection. This yields a significantly blurred shadow of the absorber in both Rayleigh and Mie-type media. The linear combination $\Phi_{41}+\Phi_{44}$ represents the shadow for the measured Stokes component $V$, and an incident right-handed circularly polarized source. This signal is related to photons that have preserved their right-handed helicity and, in agreement with previous studies [26,36], this image is sharper in the case of the Rayleigh medium [Panel (b)] due to the rapid randomization of polarization for the photons that propagate off axis (particularly, over long optical paths). This means that this component of polarization filters out the non-ballistic trajectories. Similar results are obtained for linear polarizations in the Rayleigh case (not shown here). For the Mie scattering medium, the elements relating to the circular-polarized component [Panel (d)] appear to be almost identical to that of the unpolarized case. A similar result was found in [26] for a medium containing large particles, and it was explained by the effect of circular polarization memory $[21,22,42,43]$, which preserves the helicity of incident light over significant distances in such media, even if propagating off axis. Thus, for media having intrinsically high scattering asymmetry, measurements involving the circular component of polarization alone are not likely to significantly improve the resolution of buried objects compared to polarizationinsensitive measurements, unless the medium contains particles that can destroy circular polarization memory while maintaining a high asymmetry [44].

The data in Figs. 2(a)-2(d) are presented in a fashion similar to the earlier investigations mentioned above, where shadows of buried objects are observed with various polarization filters. However, due to the way in which we have formulated the problem, we can gain further insight by investigating what is happening within the medium. In Figs. 2(e)-2(h), we display the elements of the sensitivity kernel, $K$, computed in the object plane, that is, at $z=L / 2$, for a fixed source-detector pair positioned in the center of the field of view. These functions were used to produce the shadows of the absorber presented in Figs. 2(a)-2(d), where they are convoluted with the buried object during the scanning process to provide the images we have just discussed. Thus, the closer to a delta function the dependence of these kernel elements on $(x, y)$ is within the object plane, the sharper the shadow of an absorber will be at the detector. For the Rayleigh case, it can be seen that, while the unpolarized element $K_{11}$ is quite broad, the circular component $K_{41}+K_{44}$ is much more localized near the axis of the source-detector pair [Panels (e) and (f)]. In the Mie case, the unpolarized and the circularly polarized kernel elements are equally broad [Panels (g) and (h)]. While this result is not unexpected [26,36], our ability to efficiently compute the sensitivity kernel elements at the object location will provide valuable insight into how different linear combinations of polarization measurements can better resolve the embedded absorber, as we will see throughout the remainder of this paper.

In the next demonstration shown in Fig. 3, we consider the same Mie-scattering medium as above, but use various linear polarization filters. As in the previous example, the upper row of Fig. 3 shows the matrix elements of $\Phi$ measured for a buried object shaped as the letter "F," and the lower row shows the corresponding elements of $K$ in the object plane at $z=L / 2$. Note that the two figures for the unpolarized case [Panels (a) and (e)] are identical to the previous Mie example, and are repeated here for direct comparison. The matrix elements related to linear polarization channels are shown in Panels (b) and (c) for $\Phi$ and in Panels (f) and (g) for $K$. Here we display the linear combinations $\Phi_{21}+\Phi_{22}$ and $\Phi_{21}-\Phi_{22}$ and the corresponding combinations for $K$, which are relevant to imaging with the Stokes $Q$ component. For example, a measurement with the incident beam linearly polarized along the $X$-axis and a linear filter in front of the detector which is fully transmissive to $X$-polarized light will yield the combination $\frac{1}{2}\left[\Phi_{11}+\Phi_{12}+\Phi_{21}+\Phi_{22}\right]$ (linear co-polarized channel). If the detector is rotated to be fully transmissive to $Y$-polarized light, the measurement will yield $\frac{1}{2}\left[\Phi_{11}+\Phi_{12}-\Phi_{21}-\Phi_{22}\right]$ (linear cross-polarized channel). Subtraction of these two channels results in the shadow of the $Q$ component of the Stokes vector for $X$-polarized input, $\Phi_{21}+\Phi_{22}$. Performing a similar set of measurements, but with the incident $Y$-polarized light yields the combination $\Phi_{21}-\Phi_{22}$. Now, looking at the images in Panels (b) and (c), we see that these are blurred in an asymmetric fashion, which mirrors the asymmetry in the corresponding images in Panels ( $\mathrm{f}$ ) and (g). However, when the latter two images are summed together, resulting in the image shown in Panel (d), the asymmetry is reduced and the object becomes more visible. This is due to the increase in sharpness of the resulting kernel element $K_{21}$, as can be seen in Panel (h). The lower magnitude azimuthal features of $K_{21}$ can be seen to produce some artifacts in Panel (d), yet the outline of the target is still clearly visible. To obtain the image in Panel (d) physically, we must perform the measurement of the Stokes component $Q$ when the incident light is linearly polarized in the $X$ direction (involving two physical measurements), perform a separate measurement for incident linear polarization along $Y$ (involving two physical measurements), and sum the two images together. Thus, although this imaging modality does require multiple measurements and some post-processing of data, it can still be considered direct, as it does not involve solution of an ill-posed inverse problem. With this demonstration, it becomes clear that if we can find some linear combination of kernel elements that produce a sharp point spread function in the object plane, we can improve the sharpness of the corresponding linear combination of the data matrix elements. As a consequence, the visibility of the buried object is then 


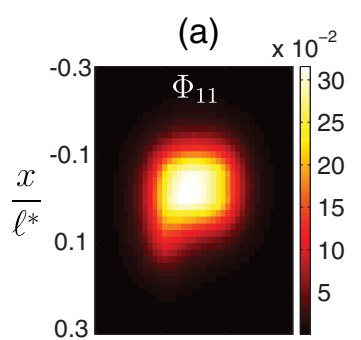

(e) (b) $\times 10^{-3}$

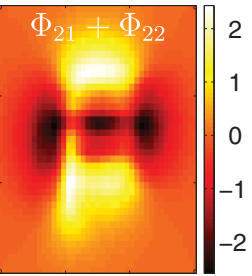

(f) (c)

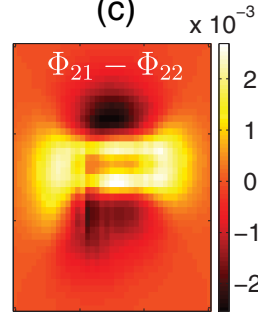

(g)

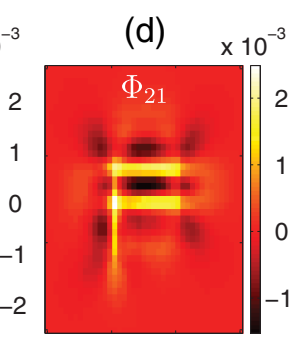

(h)
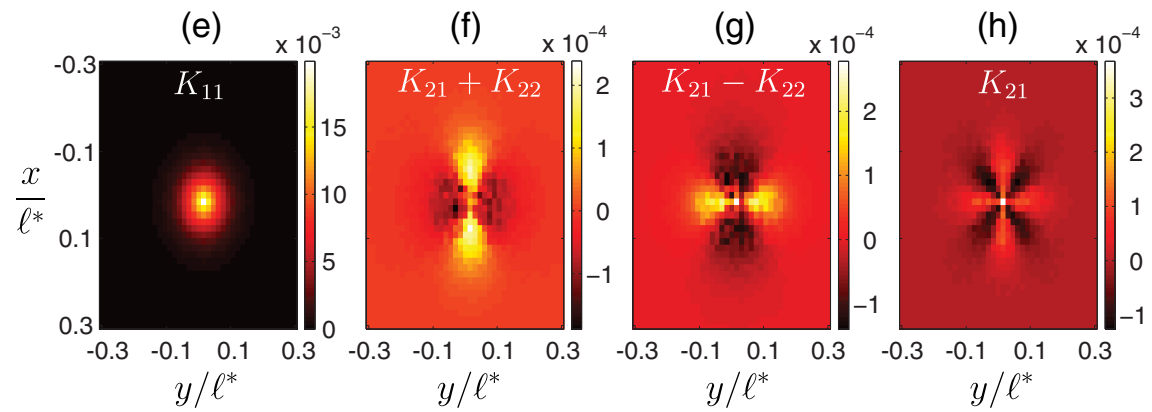

F3:1

F3:2

F3:3

Fig. 3. Elements of the matrix data function $\Phi$ [top row, Panels (a-d)] for a buried object shaped as the letter "F" obtained by scanning the sourcedetector pair in the $X Y$-plane. Corresponding elements of the sensitivity kernel [bottom row, Panels (e)-(h)] within the object plane, at $z=L / 2$, for a single, centrally located position of the source and detector. All figures are normalized to the kernel element $K_{11}$ summed over the entire object plane. Scattering medium consists of Mie-type scattering particles with asymmetry parameter $g=0.95$, and an optical depth of $L=1 \ell^{*}$.

improved. We will see more examples of this in the backscattering geometry in the next section.

One should keep in mind that the use of polarizing elements results in signals of relatively lower intensity as compared to unpolarized analysis. To elaborate on the feasibility of the various imaging channels discussed above, we have computed the ratio $\rho=\left(K_{21}+K_{22}\right) / K_{11}$ for slabs of various thickness $L$. The quantity $\rho$, similar to a degree of polarization, but of the shadow of an inhomogeneity, is computed in the central pixel (on the source-detector axis) and in the mid-plane of the slab, $z=L / 2$. The dependence of $\rho$ on the slab width $L$ is shown in Fig. 4. As expected, $\rho$ decreases rapidly with $L$. If we consider the ratio $\rho=0.01$ as the limit of detectability of the signal (which assumes we can observe a shadow 2 orders

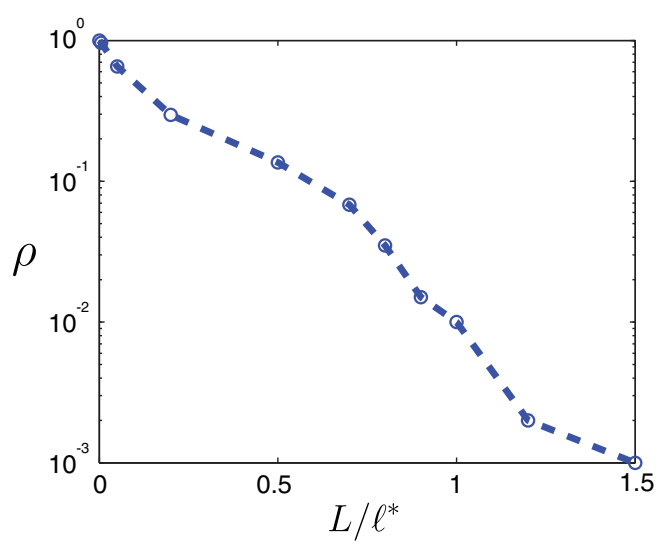
and source and detector arranged on axis in the transmission geometry. Medium consists of Mie particles with scattering asymmetry $g=0.95$. of magnitude lower than the unpolarized shadow), then the total slab thickness after which the use of linear polarization filters is no longer possible is $\sim \ell^{*}$ for this Mie medium (with $g=0.95)$ in the transmission geometry.

\section{SCANNING IN THE BACKSCATTERING GEOMETRY}

In this section we investigate the sensitivity to buried objects as measured by a scanning source-detector pair in the backscattering configuration. This geometry is of particular interest in applications involving biomedical imaging, as it is most suitable for non-invasive monitoring of superficial layers of soft tissues. Referring to Fig. 1(b), a collimated source is normally incident on the medium surface and a collimated detector is arranged to collect light exiting at some distance from the source and in the direction $\hat{\mathbf{s}}_{b}$. This arrangement is held fixed while the sourcedetector pair is scanned across the medium surface. In this example, we consider a medium with similar properties to that of Intralipid, which is a common phantom material used to approximate scattering in biological tissues. The medium used in the simulation consists of a polydispersion of spheres with an exponential distribution in size and the refractive index contrast $n_{s} / n_{b}=1.11$. The resulting scattering asymmetry parameter of this medium is $g=0.75$ at the wavelength $\lambda=633 \mathrm{~nm}$, which is in agreement with previously measured Intralipid properties [45]. Additionally, the scattering albedo was set to $\bar{\mu}_{s} / \bar{\mu}_{t}=0.99$, the same as in the transmission geometry.

We first display in Fig. 5 the sensitivity kernel elements in the object plane (at $z=L / 2$ ) for the detection angle of $\theta=$ $10^{\circ}$ to the normal, a source-detector separation of $0.32 \ell^{*}$, and a total slab thickness of $L=3.6 \ell^{*}$. In this configuration, the axis of the incident source and the axis of the detector intersect in the object plane at $z=1.8 \ell^{*}$. This arrangement is chosen to 


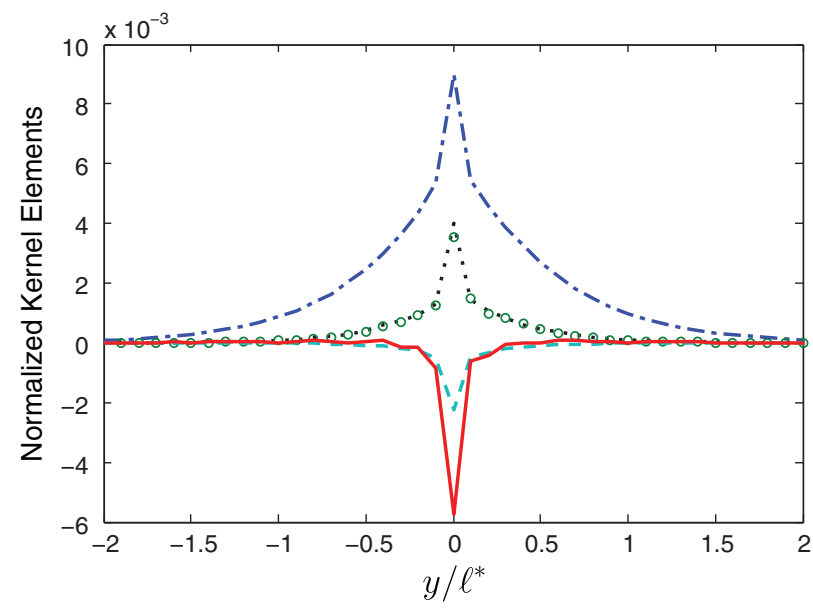

F5:1 Fig. 5. Sensitivity kernel elements along the $Y$ direction within the F5:2 object plane. Kernel element $K_{11}$ (dotted-dashed blue). Kernel F5:3 element $2 K_{22}$ (circles green). Kernel element $2 K_{33}$ (black dots). F5:4 Kernel elements $K_{41}+K_{44}$ (cyan dashed). Kernel elements F5:5 $\frac{1}{2}\left[2 K_{22}+2 K_{33}+8.5\left(K_{41}+K_{44}\right)\right]$ (solid red). All elements are norF5:6 malized to $K_{11}$ summed over the entire object plane. maximize the effect of a perturbation on the single scattering contribution at the detector. If, instead, the axes are not overlapping at the object location, the scanning process will result in two copies of the buried object (with a spacing directly relating to the source-detector misalignment in the object plane) or, for deeper objects, the result is a significant increase in blurring, with the features of the object being elongated in the direction of the source-detector separation.

In Fig. 5, various sensitivity kernel elements are plotted as functions of the coordinate $y$ for $x=0$ and $z=L / 2$. We display the elements in this case as one-dimensional scans so that we can see small details in the functions more clearly. The $K_{11}$ element, corresponding to unpolarized illumination and detection, can be seen to have the most broad distribution, as was also the case in the transmission geometry. A central peak can still be observed at the point where the source and detector intersect. This is due to single scattering, which dominates for sufficiently shallow locations (given that the detector conditions allow it), as can be shown analytically [46]. The central peak of the $K_{41}+K_{44}$ kernel elements, corresponding to the Stokes $V$-component and incident right-handed circularly polarized light, is seen to be negative. This is expected because single scattering at a large scattering angle $\left(170^{\circ}\right.$ in this case) results in a flip of helicity for this particular medium. All such elements relating to polarized contributions can also be seen to have significantly sharper peaks than the unpolarized $K_{11}$ element, suggesting the effective gating of single-scattered photons. These other elements, however, still exhibit some broad, low-magnitude tails, which are due to multiply scattered photons. The tails naturally tend to blur the image recorded by scanning the source-detector pair. Therefore, we wish to find some linear combination of these curves that corresponds to the central peak being as close to a delta function as possible. We were able to find that, for this medium, the linear combination $2 K_{22}+2 K_{33}+8.5\left(K_{41}+K_{44}\right)$ (shown by the solid red line in the figure) can achieve just that.

To verify that the corresponding combination of the elements of $\Phi$ increases the resolution of a buried object, we compare the corresponding matrix data elements to other linear combinations. In Fig. 6, we show these various data matrix elements for a buried object, this time in the shape of a cross. Here we see in Panel (b) that the $\Phi_{11}$ element, relating to polarization-insensitive imaging, is again the most blurred case. Panels (c)-(f), relating to various linear polarization channels, show a small improvement relative to the unpolarized case, yet each exhibits a noticeable asymmetry, which can skew the interpretation of the target. Panel (f), which results from

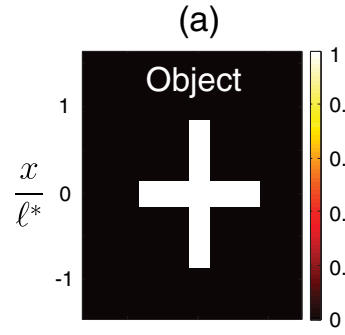

(e)

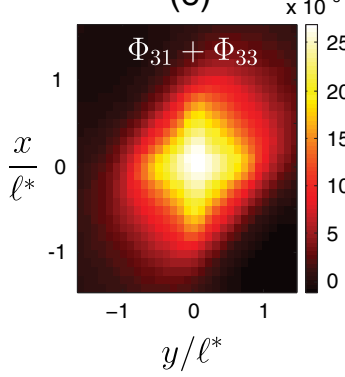

(b)

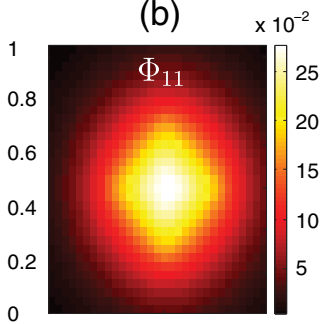

(f)

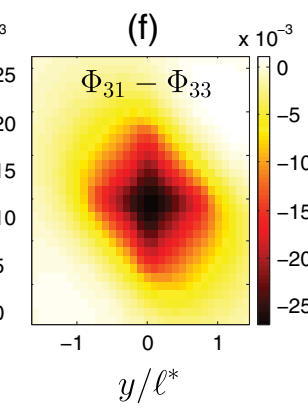

(c)

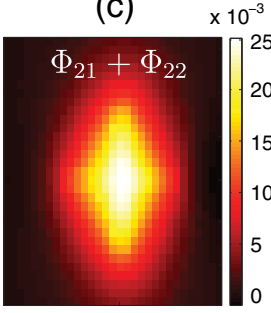

(g)

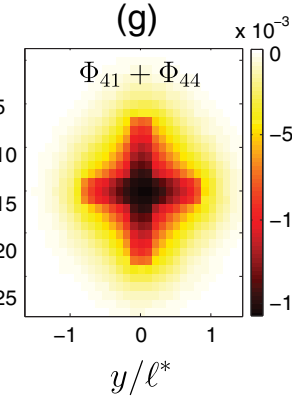

(d)

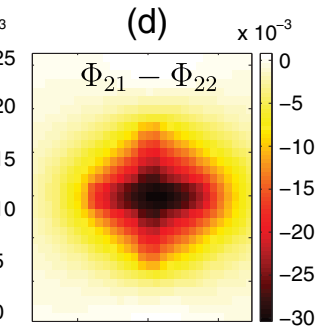

(h)

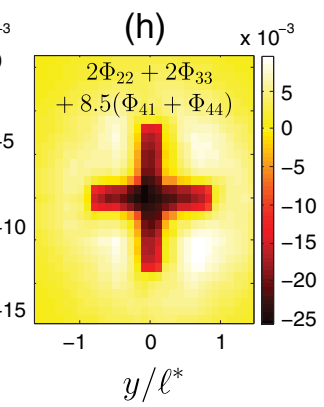

F6:1

F6:2

Fig. 6. Various linear combinations of the data matrix elements $\Phi$, measured in the backscattering geometry for a cross-shaped target located in the object plane at the depth $z=1.8 \ell^{*}$. Panel (a) shows the object shape. All matrix elements are normalized to $K_{11}$ summed over the entire object plane. 
circular polarization channels, shows a higher symmetry and a somewhat improved level of visibility of the object. However, as predicted, the case with the greatest visibility of the buried absorber is that of the combination $2 \Phi_{22}+2 \Phi_{33}+8.5\left(\Phi_{41}+\Phi_{44}\right)$, which is shown in Panel (h). In addition to a sharp peak in the object plane, the corresponding linear combination of sensitivity kernel elements also results in a highly symmetric point spread function in the object plane. To physically attain the linear combination shown in Panel (h), a series of 10 different measurements must be performed (for both the background medium and the medium containing the target). However, to put this in more simple terms, the image in Panel (h) is simply obtained by combining the images in other panels according to the rule (c)-(d) $+(\mathrm{e})-(\mathrm{f})+8.5(\mathrm{~g})$. We found that, for the considered medium type and the detection angle of $10^{\circ}$, this linear combination of the $K$ components was the most effective for a range of slab thicknesses, where the example shown with $L=3.6 \ell^{*}$ was toward the upper limit for which a clear direct image of the object was still visible.

\section{SUMMARY AND DISCUSSION}

In this work, we have used the efficient numerical tools recently developed in [15] to demonstrate the potential of polarization filtering in the context of imaging through turbid media.

While not all of the results shown are surprising (e.g., using circular or linear polarization in transmission through Rayleightype media $[26,36])$, we have gone a step further than simply using polarized light for illumination and polarization filters for detection. First, our simulations involve a collimated sourcedetector pair scanned over the surface of a plane-parallel medium either in the transmission geometry (on axis) or in the reflection geometry. Second, to achieve the best images, we analyze the sensitivity kernel, $K$, within the medium, at the location of the buried object. This informs us on which post-processing of the scan data recorded via several physical measurement channels is required. For example, to achieve the result shown in Fig. 6(h), 10 separate scans should be performed for both the background (reference) medium and the medium containing the target.

The task of determining the optimal combination of the physical measurement channels is non-trivial and the simulation technique developed in [15] can help seeking such combinations. One important result is that the choice of the optimal combinations depends strongly on the types of medium and on the imaging geometry. In the transmission geometry, with onaxis imaging of media containing optically large particles characterized by forward-peaked Mie scattering, the use of linear polarization filters is optimal. We have found that the best linear combination of channels is achieved by measuring the difference in intensity between cross-polarized and co-polarized channels (Stokes $Q$ component). In other words, we first illuminate the medium by an $X$-polarized beam and perform two scans with $X$ - and $Y$-polarized linear filters at the detector. Then the same two scans are repeated for the $Y$-polarized incident beam, requiring four separate scans in total. We emphasize that the data recorded in these four scans are not redundant, as is clearly demonstrated by comparing
Figs. 3(b) and 3(c). In the case of backreflection geometry for an Intralipid-like medium, discussed in Section 4, we found that 10 independent scans involving linear and circular polarization filters are required to achieve the optimal result.

With reference to media containing optically small Rayleigh scatterers, these are in some sense easier to image with polarization gating, especially in the transmission geometry, due to the rapid polarization randomization of non-ballistic photons. Our results indicate that polarization gating can be useful in such media up to the depth of $\sim 20 \ell^{*}$. In this case, obtaining depth resolution is also feasible by varying the angles of incidence and detection. Additionally, the problem of backscattering in such media is similar to the problem of inverting the broken-ray transform [46]. What is achieved by polarization gating is the increased precision of the broken-ray transform description, which relies on detection of singly scattered light. Inversion of the broken-ray transform is possible if many scans are performed with different source-detector separations, so that some depth resolution can be achieved.

In summary, by manipulating the contribution of the various sensitivity kernel elements in the object plane via simple linear combinations, we can find the optimal set of physical measurements and post-processing of the recorded data that will result in the clearest image of the buried absorber. This technique offers a more rational approach toward customizing a polarization-filtering scheme for a given medium and imaging geometry than simply trialling large numbers of physical measurements with no information as to the effect this can have on the resulting images.

Funding. Agence Nationale de la Recherche (ANR) (ANR- 1 11-IDEX-0001-02).

Acknowledgment. This work has been carried out thanks to the support of the $A^{*}$ MIDEX project funded by the "Investissements d'Avenir" French Government program, managed by the French National Research Agency (ANR).

\section{REFERENCES}

1. M. O'Leary, D. Boas, B. Chance, and A. Yodh, "Refraction of diffuse photon density waves," Phys. Rev. Lett. 69, 2658-2661 (1992).

2. T. Durduran, R. Choe, W. B. Baker, and A. G. Yodh, "Diffuse optics for tissue monitoring and tomography," Rep. Prog. Phys. 73, 076701 (2010).

3. S. R. Arridge, "Optical tomography in medical imaging," Inverse Probl. 15, R41-R93 (1999).

4. D. A. Boas, D. H. Brooks, E. L. Miller, C. A. DiMarzio, M. Kilmer, R. J. Gaudette, and Q. Zhang, "Imaging the body with diffuse optical tomography," IEEE Signal Process. Mag. 18, 57-75 (2001).

5. S. R. Arridge and J. C. Schotland, "Optical tomography: forward and inverse problems," Inverse Probl. 25, 123010 (2009).

6. R. Choe, A. Corlu, K. Lee, T. Durduran, S. Konecky, S. Arridge, B. Czerniecki, D. Fraker, A. DeMichele, B. Chance, and A. Yodh, "Diffuse optical tomography of breast cancer during neoadjuvant chemotherapy: a case study with comparison to MRI," Med. Phys. 32, 1128-1139 (2005).

7. R. Choe, S. D. Konecky, A. Corlu, K. Lee, T. Durduran, D. R. Busch, B. J. Czerniecki, J. Tchou, D. L. Fraker, A. DeMichele, B. Chance, S. R. Arridge, M. Schweiger, J. P. Culver, M. D. Schnall, M. E. Putt, M. A. Rosen, and A. G. Yodh, "Differentiation of benign and malignant breast tumors by in-vivo three-dimensional parallel-plate diffuse optical tomography," J. Biomed. Opt. 14, 024020 (2009). 
8. W. Cai, M. Lax, and R. R. Alfano, "Cumulant solution of the elastic Boltzmann transport equation in an infinite uniform medium," Phys. Rev. E 61, 3871-3876 (2000).

9. W. Cai, M. Lax, and R. R. Alfano, "Analytical solution of the polarized photon transport equation in an infinite uniform medium using cumulant expansion," Phys. Rev. E 63, 016606 (2000).

10. M. Xu, W. Cai, M. Lax, and R. R. Alfano, "Photon migration in turbid media using a cumulant approximation to radiative transfer," Phys. Rev. E 65, 066609 (2002).

11. V. A. Markel, "Modified spherical harmonics method for solving the radiative transport equation," Waves Random Complex Media 14, L13-L19 (2004).

12. G. Panasyuk, J. C. Schotland, and V. A. Markel, "Radiative transport equation in rotated reference frames," J. Phys. A 39, 115-137 (2006).

13. M. Machida, G. Y. Panasyuk, J. C. Schotland, and V. A. Markel, "The Green's function for the radiative transport equation in the slab geometry," J. Phys. A 43, 065402 (2010).

14. M. Machida, "An $F_{N}$ method for the radiative transport equation in three dimensions," J. Phys. A 48, 325001 (2015)

15. U. Tricoli, C. M. Macdonald, A. Da Silva, and V. A. Markel, "Reciprocity relation for the vector radiative transport equation and its application to diffuse optical tomography with polarized light," Opt. Lett. 42, 362-365 (2017).

16. G. S. Abdoulaev and A. H. Hielscher, "Three-dimensional optical tomography with the equation of radiative transfer," J. Electron. Imaging 12, 594-601 (2003).

17. A. Joshi, J. C. Rasmussen, E. M. Sevick-Muraca, T. A. Wareing, and J. McGhee, "Radiative transport-based frequency-domain fluorescence tomography," Phys. Med. Biol. 53, 2069-2088 (2008).

18. G. Bal, "Inverse transport theory and applications," Inverse Probl. 25 , 053001 (2009)

19. M. Machida, G. Y. Panasyuk, Z.-M. Wang, V. A. Markel, and J. C. Schotland, "Radiative transport and optical tomography with large datasets," J. Opt. Soc. Am. A 33, 551-558 (2016).

20. M. Xu and R. R. Alfano, "Random walk of polarized light in turbid media," Phys. Rev. Lett. 95, 213901 (2005).

21. F. C. MacKintosh, J. X. Zhu, D. J. Pine, and D. A. Weitz, "Polarization memory of multiply scattered light," Phys. Rev. B 40, 8342-8348 (1989).

22. M. Xu and R. R. Alfano, "Circular polarization memory of light," Phys. Rev. E 72, 065601 (2005).

23. J. D. van der Laan, D. A. Scrymgeour, S. A. Kemme, and E. L. Dereniak, "Detection range enhancement using circularly polarized light in scattering environments for infrared wavelengths," Appl. Opt. 54, 2266-2274 (2015).

24. M. Dubreuil, P. Delrot, I. Leonard, A. Alfalou, C. Brosseau, and A. Dogariu, "Exploring underwater target detection by imaging polarimetry and correlation techniques," Appl. Opt. 52, 997-1005 (2013).

25. S. G. Demos and R. R. Alfano, "Optical polarization imaging," Appl. Opt. 36, 150-155 (1997).

26. J. M. Schmitt, A. H. Gandjbakhche, and R. F. Bonner, "Use of polarized light to discriminate short-path photons in a multiply scattering medium," Appl. Opt. 31, 6535-6546 (1992).

27. O. Emile, F. Bretenaker, and A. Le Floch, "Rotating polarization imaging in turbid media," Opt. Lett. 21, 1706-1708 (1996).
28. S. Mujumdar and H. Ramachandran, "Imaging through turbid media using polarization modulation: dependence on scattering anisotropy," Opt. Commun. 241, 1-9 (2004).

29. M. P. Silverman and W. Strange, "Object delineation within turbid media by backscattering of phase-modulated light," Opt. Commun. 144, 7-11 (1997).

30. G. D. Lewis, D. L. Jordan, and P. J. Roberts, "Backscattering target detection in a turbid medium by polarization discrimination," Appl. Opt. 38, 3937-3944 (1999).

31. X. Ni, Q. Xing, W. Cai, and R. R. Alfano, "Time-resolved polarization to extract coded information from early ballistic and snake signals through turbid media," Opt. Lett. 28, 343-345 (2003).

32. D. A. Miller and E. L. Dereniak, "Selective polarization imager for contrast enhancements in remote scattering media," Appl. Opt. 51, 40924102 (2012).

33. A. da Silva, C. Deumié, and I. Vanzetta, "Elliptically polarized light for depth resolved optical imaging," Biomed. Opt. Express 3, 2907-2915 (2012).

34. S. Sridhar and A. Da Silva, "Enhanced contrast and depth resolution in polarization imaging using elliptically polarized light," J. Biomed. Opt. 21, 071107 (2016)

35. J. S. Tyo, "Enhancement of the point-spread function for imaging in scattering media by use of polarization-difference imaging," J. Opt. Soc. Am. A 17, 1-10 (2000).

36. M. Moscoso, J. B. Keller, and G. Papanicolaou, "Depolarization and blurring of optical images by biological tissues," J. Opt. Soc. Am. A 18, 948-960 (2001).

37. A. Ishimaru and C. W. Yeh, "Matrix representations of the vector radiative-transfer theory for randomly distributed nonspherical particles," J. Opt. Soc. Am. A 1, 359-364 (1984).

38. J. C. Schotland and V. A. Markel, "Fourier-Laplace structure of the inverse scattering problem for the radiative transport equation," Inverse Probl. Imaging 1, 181-188 (2007).

39. G. Y. Panasyuk, V. A. Markel, and J. C. Schotland, "Superresolution and corrections to the diffusion approximation in optical tomography," Appl. Phys. Lett. 87, 101111 (2005)

40. C. F. Bohren and D. R. Huffman, Absorption and Scattering of Light by Small Particles (Wiley, 2008).

41. K. M. Yoo, F. Liu, and R. R. Alfano, "Imaging objects hidden in scattering media using an absorption technique," Opt. Lett. 16, 1068-1070 (1991).

42. W. Cai, X. Ni, S. K. Gayen, and R. R. Alfano, "Analytical cumulant solution of the vector radiative transfer equation investigates backscattering of circularly polarized light from turbid media," Phys. Rev. E 74, 056605 (2006).

43. A. D. Kim and M. Moscoso, "Chebyshev spectral methods for radiative transfer," SIAM J. Sci. Comput. 23, 2074-2094 (2002).

44. C. M. Macdonald, S. L. Jacques, and I. Meglinski, "Circular polarization memory in polydisperse scattering media," Phys. Rev. E 91, 033204 (2015).

45. H. J. van Staveren, C. J. M. Moes, J. van Marie, S. A. Prahl, and M. J. C. van Gemert, "Light scattering in intralipid-10\% in the wavelength range of 400-1100 nm," Appl. Opt. 30, 4507-4514 (1991).

46. L. Florescu, J. C. Schotland, and V. A. Markel, "Single-scattering optical tomography," Phys. Rev. E 79, 036607 (2009). 


\section{Queries}

1. AU: The funding information for this article has been generated using the information you provided to OSA at the time of article submission. Please check it carefully. If any information needs to be corrected or added, please provide the full name of the funding organization/institution as provided in the CrossRef Open Funder Registry (http://www.crossref.org/fundingdata/registry.html).

2. AU: Please confirm the last page added to the Refs. [21,33].

3. AU: The volume has been changed from ' 92 ' to ' 31 '. Please confirm this is correct.

4. AU: Please check the edit made in the page range in Ref. [43].

\section{ORCID Identifiers}

The following ORCID identification numbers were supplied for the authors of this article. Please review carefully. If changes are required, or if you are adding IDs for authors that do not have them in this proof, please submit them with your corrections for the article.

- Vadim A. Markel https://orcid.org/0000-0002-9748-6865 